\section{Surgical treatment and optical coherence tomographic evaluation for accidental laser-induced full-thickness macular holes}

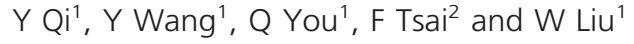

${ }^{1}$ Beijing Tongren Eye Center, Beijing Ophthalmology and Visual Science Key Lab, Beijing Tongren Hospital, Capital Medical University, Beijing, China

${ }^{2}$ Sharp Rees-Stealy Medical Group, San Diego, CA, USA

Correspondence: W Liu, Beijing Tongren Eye Center, Beijing Ophthalmology and Visual Science Key Lab, Beijing Tongren Hospital, Capital Medical University, 1 Dongjiaomminxiang Street, Dongcheng District, Beijing 100730, China Tel: +86 58269157; Fax: +8658269159.

E-mail: wuliubj@yahoo.com

Received: 26 August 2016 Accepted in revised form: 25 January 2017

Published online: 10 March 2017

\begin{abstract}
Purpose To report OCT appearance and surgical outcomes of full-thickness macular holes (MHs) accidentally caused by laser devices.

Patients and methods This retrospective case series included 11 eyes of 11 patients with laser-induced MHs treated by pars plana vitrectomy, internal limiting membrane (ILM) peeling, and gas or silicone oil tamponade. Evaluations included a full ophthalmic examination, macular spectral-domain optical coherence tomography (SD-OCT), and fundus photography. Main outcome measures is $\mathrm{MH}$ closure and final visual acuity; the secondary outcome was the changes of retinal pigment epithelium and photoreceptor layer evaluated by sequential post-operative SD-OCT images. Results Five patients were accidentally injured by a yttrium aluminum garnet (YAG) laser and six patients by handheld laser. MH diameters ranged from 272 to $815 \mu$ m (mean, $505.5 \pm 163.0 \mu \mathrm{m})$ preoperatively. Bestcorrected visual acuity (BCVA) improved from a mean of $0.90 \operatorname{logMAR}$ (range, counting finger-8/20) preoperatively to a mean of 0.34 logMAR (range, a counting finger-20/20) postoperatively $(P=0.001, t=4.521)$. Seven of 11 patients $(63.6 \%)$ achieved a BCVA better than 10/20. Ten patients had a subfoveal hyperreflectivity and four patients had a focal choroidal depression subfoveal preoperatively. At the last follow-up, all 11 eyes demonstrated the following: closure of the macular hole, variable degrees of disruption of external limiting membrane (ELM) and outer photoreceptor ellipsoid and interdigitation bands. In 10 eyes, the disruption was in the form of focal defects in the outer retina. After surgery, the subfoveal

hyperreflectivity and focal choroidal depression remained.

Conclusion Accidental laser-induced fullthickness macular holes can be successfully closed with surgery. Inadvertent retinal injury from laser devices, especially handheld laser injury has occurred with increasing frequency in recent years. However, there is a paucity of data regarding these types of injuries, mostly in the form of case reports. We hereby reported 11 eyes of 11 patients with laserinduced macular holes treated by vitrectomy. All the macular holes closed after surgery and the corresponding visual acuities significantly improved postoperatively.

Eye (2017) 31, 1078-1084; doi:10.1038/eye.2017.41; published online 10 March 2017

\section{Introduction}

A laser-induced macular hole is a special type of traumatic macular hole. Although not frequently seen clinically, it may influence patients' visual acuities dramatically. Inadvertent retinal injury from laser devices have occurred with increasing frequency in recent years. ${ }^{1-3}$

Laser-induced macular holes have been reported in previous articles. ${ }^{4-7}$ Neodymiumdoped yttrium aluminum garnet (Nd:YAG) lasers (including titanium-sapphire lasers) and handheld lasers were major reasons for laserinduced macular holes in previous reports. ${ }^{4-10}$ Most of previous reports were case reports. ${ }^{4-10}$ We collected a relatively large case series of 11 eyes of 11 consecutive patients with laserinduced macular holes treated with vitrectomy at Beijing Tongren Hospital, Beijing, China, to study the anatomic and visual outcomes of these patients.
\end{abstract}




\section{Materials and methods}

This retrospective, non-comparative, interventional case series included all 11 patients who presented with decreased visual acuity due to laser-induced macular holes and who underwent vitrectomy in the Eye Center of Beijing Tongren Hospital from September 2010 to March 2016. The Medical Ethics Committee of the Beijing Tongren Hospital approved the study protocol, and all participants gave their informed consent. All patients underwent a regular ophthalmological examination including refraction with an assessment of best-corrected visual acuity (BCVA), fundus photography (fundus camera, TRC-50; Topcon, Tokyo, Japan), and spectraldomain optical coherence tomography (SD-OCT) of the macula (Stratus OCT; Carl Zeiss Meditec, Dublin, CA, USA).

A standard 23-gauge 3-port pars plana vitrectomy was performed by the same surgeon (W.L.). A core vitrectomy was performed and the internal limiting membrane was removed without staining. The posterior hyaloid was elevated and trimmed in all patients. A fluid-gas exchange was carried out, and the vitreous was filled with an inert gas (C2F6 or C3F8). For one patient with the biggest macular hole, the eye was filled with silicone oil (5000cs) for its long-term tamponade. Patients were asked to stay in a prone position for at least 1 week after surgery; the silicone oil was removed 3 months later.

At post-operative follow-ups, the measurement of BCVA and OCT were routinely performed at 1, 3, 6, and 12 months. The follow-up durations ranged from 3 to 36 months.

\section{Statistical analysis}

Statistical analysis was performed using a commercially available statistical software package (SPSS for Windows, version 21.0, IBM-SPSS, Chicago, IL, USA). BCVA measurements were converted to the logarithm of the minimum angle of resolution (LogMAR). The parameters are presented as mean \pm s.d.'s. Pre- and post-operative visual acuities were compared using paired Student's $t$-test. The correlation between the size of the macular hole and BCVA was analyzed using Pearson correlation. The correlation between the thickness of foveal residual neuroretina or subfoveal defect region and BCVA after surgery was analyzed using Spearman correlation. A $P$-value $<0.05$ was considered statistically significant.

We certify that all applicable institutional and governmental regulations concerning the ethical use of human volunteers were followed during this study.

\section{Results}

The study included 11 eyes of 11 consecutive patients. There were 3 female patients ( 3 eyes) and 8 male patients (8 eyes). Five patients were injured by an Nd:YAG laser and six patients were injured by handheld lasers. In Nd: YAG laser injured eyes, three of them were injured by an industrial laser $(P 1,3,5)$ and two of them were injured by a cosmetic laser (P 2,4). In the handheld laser injured patients, one had disruption of outer photoreceptor ellipsoid and interdigitation bands in the contralateral eye (P 9). Among all the patients, 4 were self-inflicted injuries, 5 were non-self-inflicted trauma, and 2 were unknown.

The mean age of the patients was $22.5 \pm 9.8$ years. BCVA ranged from counting finger to 8/20 (LogMAR, 2.7 to 0.4), and the minimum diameter of the macular hole ranged from $272-815 \mu \mathrm{m}$ (mean, $505.5 \pm 163.0 \mu \mathrm{m}$ ) preoperatively (Table 1). The interval from time of injury to surgery ranged from 1 to 12 months (mean, $3.9 \pm 3.9$ months). The distance of the laser device from the eye at time of trauma ranged from 0.05 to $5 \mathrm{~m}$. The presence of a full-thickness macular hole was confirmed in all eyes on SD-OCT. Cystoid changes were observed at the margin in all 11 eyes. There was no apparent macular hemorrhage upon initial presentation in the affected eyes. (Figure 1) Information about the laser's characteristics and nature of injury was not provided for two patients.

All 11 operated eyes (100\%) demonstrated closure of the macular hole at last follow-up after a single surgery. None of these patients developed visually significant post-operative cataract during follow-up. All of 11 patients were followed for 3 months or more. The mean follow-up duration was 14.7 months (range, 3-36 months). The mean BCVA in the 11 eyes improved from 0.90 LogMAR (range, LogMAR 0.4-2.7) preoperatively to 0.34 LogMAR (range, LogMAR 0-1.7; $P=0.001, t=4.521)$. Seven of 11 patients $(63.6 \%)$ achieved a BCVA better than 10/20 postoperatively. The preoperative BCVA was marginally significantly correlated with the size of macular holes $(P=0.048$, Pearson correlation $=-0.811$ ) in Nd:YAG laser injured eyes, while the same correlationship was not found in handheld laser injured eyes $(P=0.073$, Pearson correlation $=-0.668)$. The post-operative BCVA had no correlation with the pre-operative size of the macular hole for both Nd:YAG injured eyes $(P=0.078$, Pearson correlation $=-0.736)$ and handheld laser injured eyes $(P=0.079$, Pearson correlation $=-0.655$ ).

Ten patients had a subfoveal hyperreflectivity on SD-OCT preoperatively (all except P5). Four patients (P 1, 3, 4, 6) had a focal subforveal choroidal depression. On SD-OCT obtained at the last follow-up, all 11 eyes demonstrated the following: closure of the macular hole, variable degrees of disruption of external limiting 


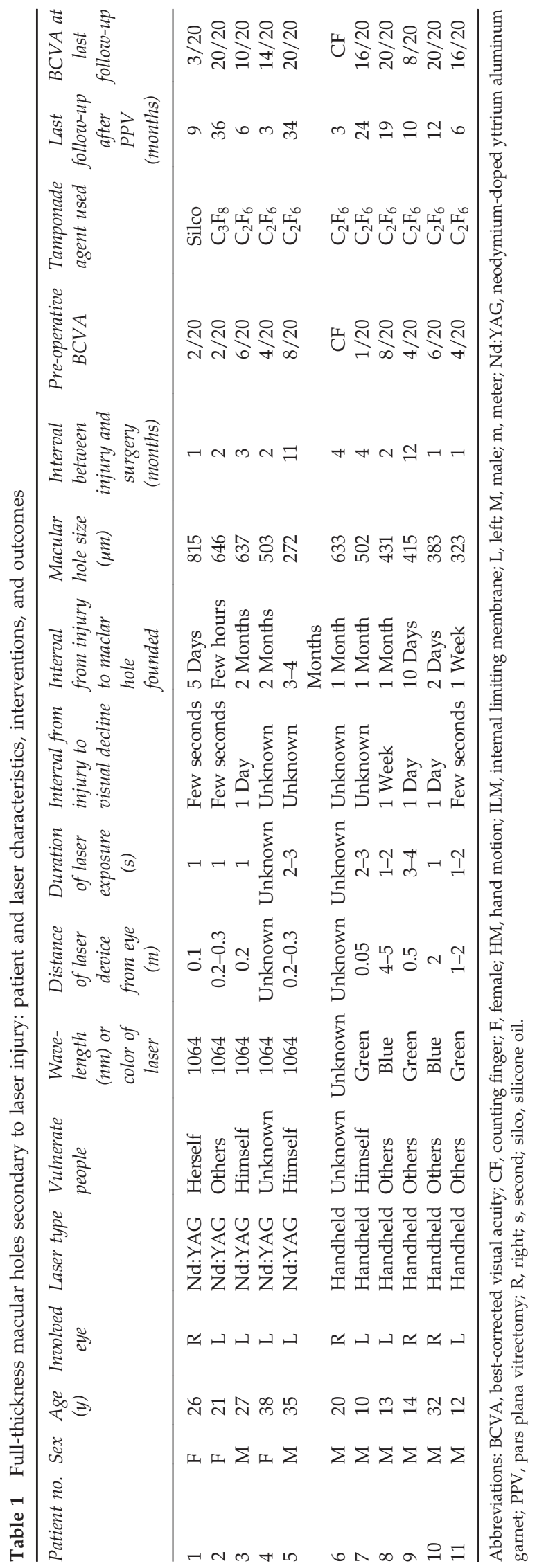

membrane and outer photoreceptor ellipsoid and interdigitation bands. In 10 of these eyes (all except P5), the disruption was in the form of a focal defect in the outer retina. After surgery, the subfoveal hyperreflectivity and focal choroidal depression remained. The focal subfoveal hyperreflectivity was consistently seen as a 'faint whitening' of the retinal pigment epithelium (RPE) on fundus examination. We consider them as the light reflex changes of retina resulting from laser injury. (Figures 1 and 2) We measured the post-operative thickness of foveal residual neuroretina and subfoveal defect region, (Table 2) and found the post-operative BCVA was significantly correlated with the former $(P=0.009$, Spearman correlation $=-0.690)$ and marginally significantly correlated with the later $(P=0.049$, Spearman correlation $=0.525$ ). No recurrence of a macular hole was noted during the follow-up period of this study.

\section{Discussion}

A laser injury may cause a full-thickness macular hole immediately after injury or after several days., ${ }^{2,9,10}$ In our case series, patients were first seen at their local hospital before being referred to us due to unimproved visual acuity, which is why our patients often had an extensive amount of time between onset of injury to presentation.

The visual prognosis in eyes with severe tissue disruption is often dependent on the distance of the retinal hole from the fovea. Although laser-induced macular holes may seal spontaneously, a typical patient who is treated conservatively will experience a severe and permanent decrease in vision. ${ }^{11-15}$ According to previous reports, spontaneous closure occurred only in macular holes with a small diameter of $<180 \mu \mathrm{m}$. Most laserinduced macular holes enlarged, and visual acuity worsened without surgery. ${ }^{16-18}$ In our case series, the average macular hole size was comparatively larger than those in previous reports. All the diameters of the macular holes were $>250 \mu \mathrm{m}$. Five out of 11 patients $(45.5 \%)$ were observed for more than 3 months after laser injury (Figure 3). We performed vitrectomies on these patients for laser-induced macular holes without spontaneous closure. Although some were not observed for a very long duration, we still performed surgery on them for patients who were eager to regain their visual function and macular anatomy.

Surgical treatment for a laser-induced macular hole was first reported by Ciulla and Topping. ${ }^{4}$ They reported a patient with a full-thickness macular hole and 20/60 visual acuity who underwent pars plana vitrectomy, membrane peeling, and gas tamponade. Visual acuity improved to $20 / 30$ at 6 months after surgery with closure of the macular hole. Potthöfer and Foerster reported an $\mathrm{Nd}$ :YAG laser-induced macular hole with a diameter of 
$300 \mu \mathrm{m}$. They performed vitrectomy and used autologous thrombocyte concentrate during surgery. The macular hole closed and visual acuity had improved from 0.16 to

P1

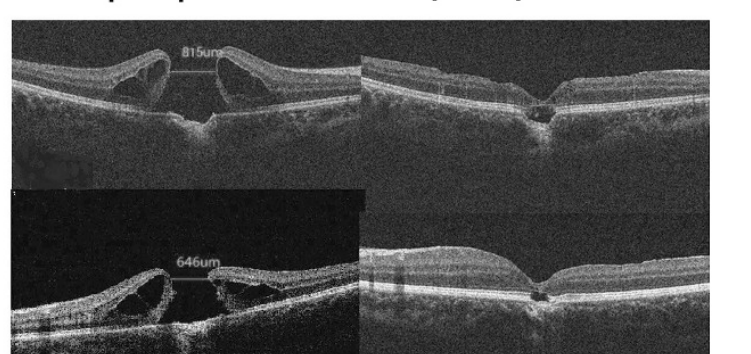

P3

P4

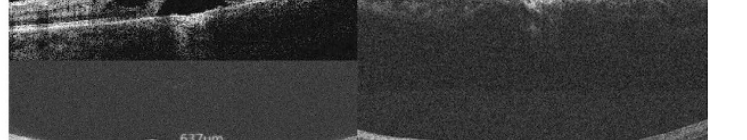

P5

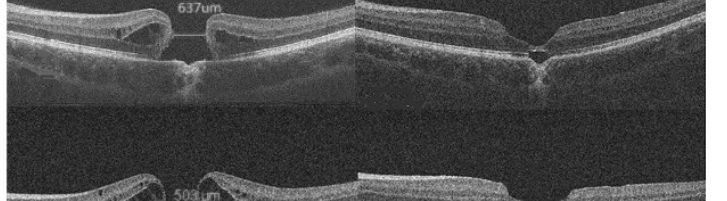

P6

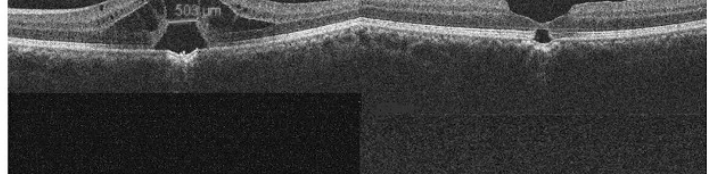

7
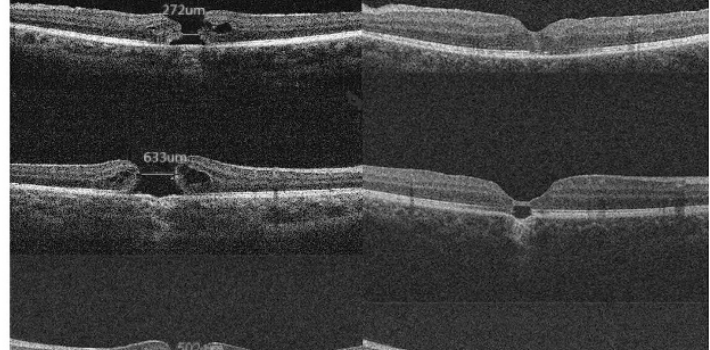

P7

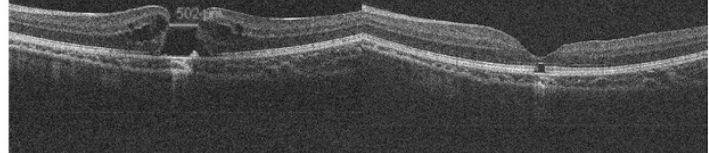

P8

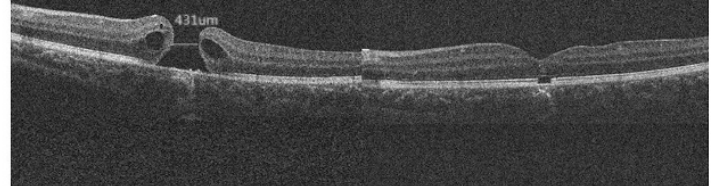

P9

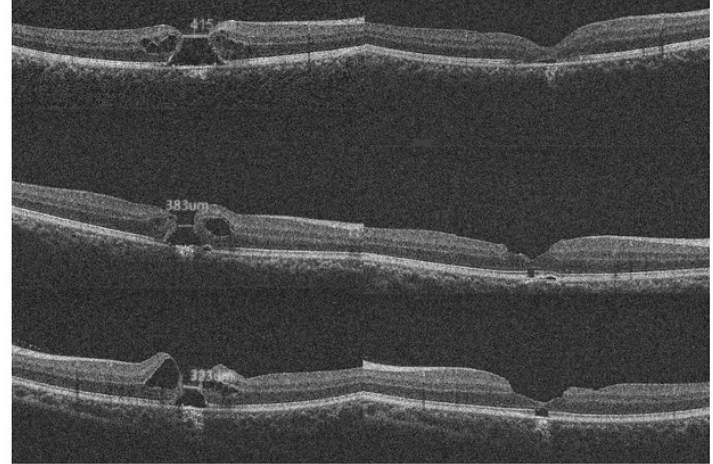

0.6 at 9 months after surgery. ${ }^{19}$ Alsulaiman et al also reported 14 eyes of 14 patients with maculopathy caused by momentary exposure to a high-power handheld blue laser; 4 patients had a full-thickness macular hole that required surgical intervention. Visual acuity improved in all patients after successful repair. ${ }^{2}$ In another report by Alsulaiman, ${ }^{18} 11$ out of 14 operated patients (78.6\%) had closure of the macular hole at final follow-up and 7 of the 11 eyes with successfully closed holes $(63.6 \%)$ achieved BCVA better than 20/40. Compared to Alsulaiman's study, in the current study, the macular hole closure rate was higher but visual prognosis was similar. These results indicate that vitrectomy is an effective treatment for laserinduced macular holes.

The macular structure disturbances observed after laser injury were diverse in different reports, and included a tardive cystic margin, opacification of the nerve fibers in Henle's layer, outer retinal defect, ellipsoid disruption, pigment hyperplasia and a subfoveal hyperreflectivity. ${ }^{19-27}$ In our cases, all patients had cystic margins, disruption of external limiting membrane and outer photoreceptor ellipsoid, and interdigitation bands. The RPE change was always in the $\mathrm{MH}$ crater in our cases. The photocoagulation-like scar was usually in the center of the $\mathrm{MH}$ crater, and the pigment excrescences were scattered throughout the MH crater at the level of RPE. Ten patients had subfoveal hyperreflectivity and four patients had a focal choroidal depression preoperatively. After surgery, the cystic margin disappeared with resolution of the macular hole, while the subfoveal hyperreflectivity and focal choroidal depression persisted. We found the patients with better post-operative BCVA had thicker foveal residual neuroretina or thinner subfoveal defect region. This suggests that the thickness of foveal residual neuroretina and subfoveal defect region might reflect the level of damage to the retina. Outer retinal defects could be found in almost all patients (10 out of 11) postoperatively. A post-operative outer retinal defect can also be found in an idiopathic macular hole. This macular structure disturbance did not seem to have any relation with visual acuity in previous reports or in our case series.

Figure 1 The figure shows the macular structures on SD-OCT of the 11 eyes before (left) and after (right) operation. (Left) 10 patients had a subfoveal hyperreflectivity on SD-OCT preoperatively (except P5). Four patients (P 1, 3, 4, 6) had a focal choroidal depression subfoveal. (Right) on SD-OCT obtained at the last follow-up, all 11 eyes demonstrated the closure of the macular hole. In 10 of these eyes (except P5), the disruption was in the form of a focal defect in the outer retina. After surgery, the subfoveal hyperreflectivity and focal choroidal depression remained. 
The patient without outer retinal defect in our report had the smallest macular hole $(272 \mu \mathrm{m})$ in the current case series; this suggested that post-operative outer retinal defect tended to occur in macular holes with large size. Determining whether it influences post-operative visual acuity requires further study.

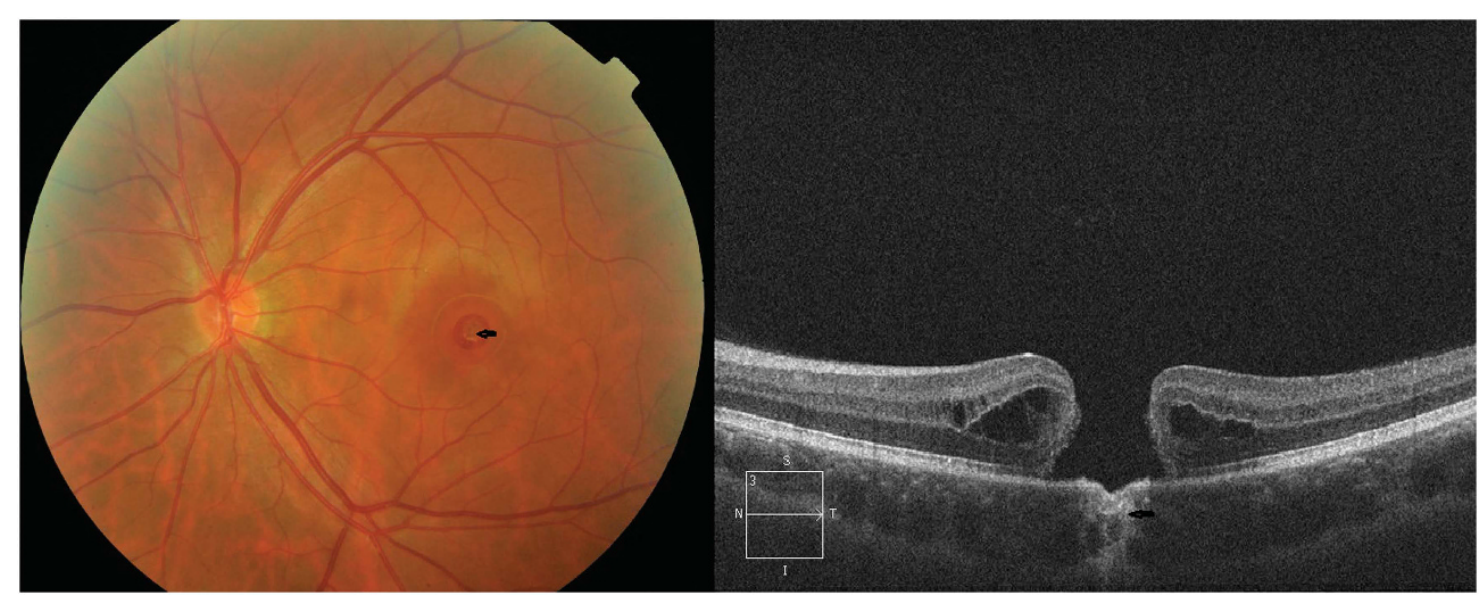

Figure 2 (Left) fundus photograph of the left eye of patient 3 showing a round full-thickness MH with faint whitening at the level of the RPE in the base of the hole (arrow). (Right) macular SD-OCT of the same patient showing a full-thickness MH and subfoveal hyperreflectivity at the level of RPE (arrow).

Table 2 The post-operative thickness of foveal residual neuroretina and subfoveal defect region

\begin{tabular}{|c|c|c|c|c|c|c|c|}
\hline Patient no. & Sex & Age (y) & Involved eye & Laser type & $\begin{array}{c}\text { BCVA at } \\
\text { last follow-up }\end{array}$ & $\begin{array}{l}\text { Thickness of foveal } \\
\text { residual neuroretina }(\mu \mathrm{m})\end{array}$ & $\begin{array}{c}\text { Thickness of subfoveal } \\
\text { defect region }(\mu \mathrm{m})\end{array}$ \\
\hline 1 & $\mathrm{~F}$ & 26 & $\mathrm{R}$ & Nd:YAG & $3 / 20$ & 52 & 148 \\
\hline 2 & $\mathrm{~F}$ & 21 & $\mathrm{~L}$ & Nd:YAG & $20 / 20$ & 81 & 74 \\
\hline 3 & $\mathrm{M}$ & 27 & $\mathrm{~L}$ & Nd:YAG & $10 / 20$ & 37 & 119 \\
\hline 4 & $\mathrm{~F}$ & 38 & $\mathrm{~L}$ & Nd:YAG & $14 / 20$ & 68 & 119 \\
\hline 5 & $\mathrm{M}$ & 35 & $\mathrm{~L}$ & Nd:YAG & $20 / 20$ & 134 & 15 \\
\hline 6 & $\mathrm{M}$ & 20 & $\mathrm{R}$ & Handheld & CF & 59 & 96 \\
\hline 7 & M & 10 & $\mathrm{~L}$ & Handheld & $16 / 20$ & 67 & 74 \\
\hline 8 & $\mathrm{M}$ & 13 & $\mathrm{~L}$ & Handheld & $20 / 20$ & 156 & 59 \\
\hline 9 & $\mathrm{M}$ & 14 & $\mathrm{R}$ & Handheld & $8 / 20$ & 104 & 44 \\
\hline 10 & $\mathrm{M}$ & 32 & $\mathrm{R}$ & Handheld & $20 / 20$ & 89 & 81 \\
\hline 11 & M & 12 & $\mathrm{~L}$ & Handheld & $16 / 20$ & 76 & 67 \\
\hline
\end{tabular}

Abbreviations: BCVA, best-corrected visual acuity; F, female; L, left; M, male; Nd:YAG, neodymium-doped yttrium aluminum garnet; R, right.

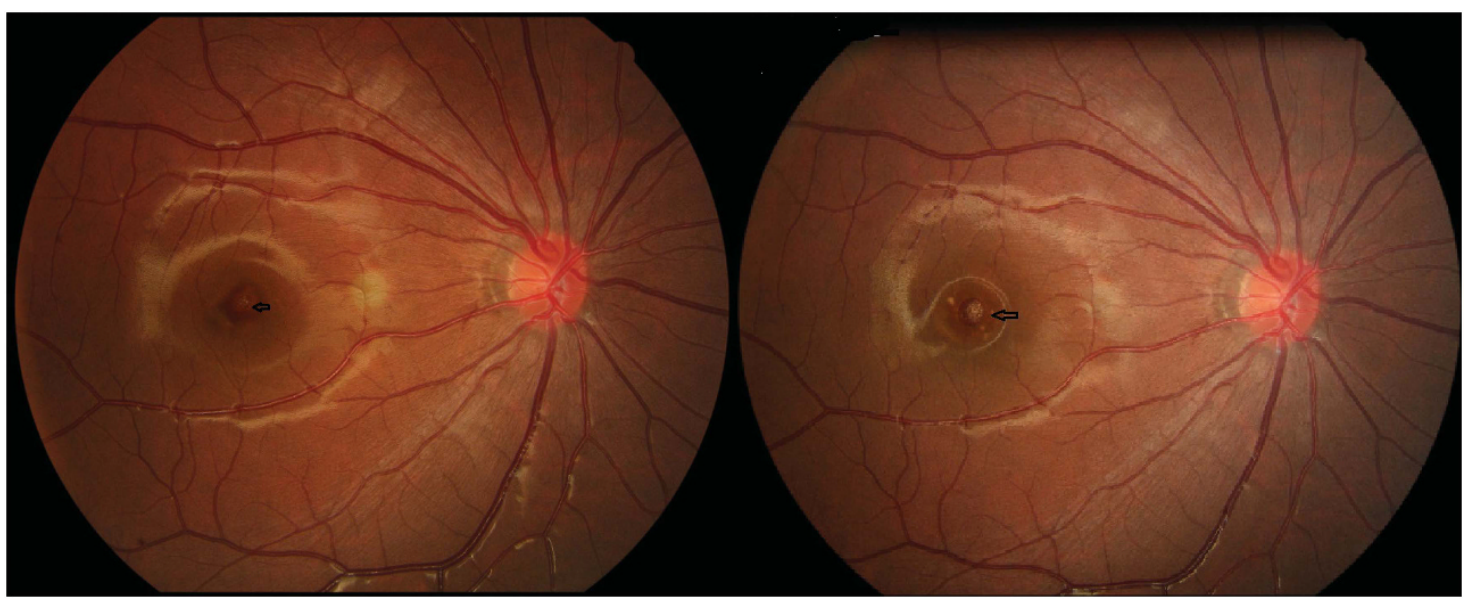

Figure 3 Fundus photographes of the right eye of patient 9 showed the MH enlarged after 1 year's observation. 
Interestingly, the pre-operative BCVA had a marginally significant correlation with the size of macular holes in Nd:YAG laser injured eyes, while the same result was not found in handheld laser injured eyes. It may be related with the mechanisms of laser injuries. The mechanism of Nd:YAG laser injury involves photomechanical and thermal tearing of the retinal tissue. Nd:YAG lasers use very short laser pulses of $1 \mu$ s or shorter. They produce mechanical side effects such as expansive microexplosions through plasma formation. Q-switched operation mode pulse durations range from a few nanoseconds to a few microseconds. As the energy is delivered in short time spans, very high peak powers of up to hundreds of megawatts can be emitted, which lead to tissue disruption. $4,5,7,8$ Handheld lasers are often used at conferences and may be used erroneously as a toy by children, which include low-power pointers and high-power handheld laser devices. High-power handheld laser devices (up to $1200 \mathrm{~mW}$ ) can affect the retina through photocoagulation. Eye injuries resulting from laser exposure are a concern because optical radiation from 380 to $1400 \mathrm{~nm}$ penetrates into various ocular structures. ${ }^{6,18}$ We considered photomechanical disruption with retinal dissolution as the pathogenesis in Nd:YAG laser-induced $\mathrm{MH}$ and photodisruptive and photothemal damage as the pathogenesis in handheld laser-induced $\mathrm{MH}$.

In conclusion, this study supported the treatment of laser-induced macular holes by vitrectomy associated with internal limiting membrane peeling and gas, or rarely, silicone oil tamponade. The pre-operative BCVA might be correlated with the size of the macular hole in Nd:YAG laser injured eyes, but did not appear to be in the case of handheld laser injured eyes. The post-operative thickness of foveal residual neuroretina and subfoveal defect region might influence the potential patients' BCVA after surgery. However, because of the small number of patients and short follow-up period of some patients, our understanding of the relationship between preoperative macular structure and post-operative visual acuity remains limited.

\section{Summary}

\section{What was known before}

- Inadvertent retinal injury from laser devices, especially handheld laser injury have occurred with increasing frequency in recent years. Although very few articles reported a case series, most were mentioned as case reports. The result of surgical treatment and the characteristic of optical coherence tomographic were still uncertain.

\section{What this study adds}

- We analyzed 11 eyes of 11 patients with laser-induced macular holes treated with vitrectomy at Beijing Tongren Hospital, Beijing, China, to study the anatomic and visual outcomes of these patients.

\section{Conflict of interest}

The authors declare no conflict of interest.

\section{References}

1 Mainster MA, Stuck BE, Brown J Jr. Assessment of alleged retinal laser injuries. Arch Ophthalmol 2004; 122(8): 1210-1217.

2 Alsulaiman SM, Alrushood AA, Almasaud J, Alzaaidi S, Alzahrani Y, Arevalo JF et al. High-power handheld blue laser-induced maculopathy: the results of the King Khaled Eye Specialist Hospital Collaborative Retina Study Group. Ophthalmology 2014; 121(2): 566-572.

3 Bhavsar KV, Wilson D, Margolis R, Judson P, Barbazetto I, Freund $\mathrm{KB}$ et al. Multimodal imaging in handheld laserinduced maculopathy. Am J Ophthalmol 2015; 159(2): 227-231.

4 Ciulla TA, Topping TM. Surgical treatment of a macular hole secondary to accidental laser burn. Arch Ophthalmol 1997; 115(7): 929-930.

5 Boldrey EE, Little HL, Flocks M, Vassiliadis A. Retinal injury due to industrial laser burns. Ophthalmology 1981; 88(2): 101-107.

6 Barkana Y, Belkin M. Laser eye injuries. Surv Ophthalmol 2000; 44(6): 459-478.

7 Goldberg MF, Young RSL, Read J, Cunha-Vaz JG. Macular hole caused by a 589-nanometer dye laser operating for 10 nanoseconds. Retina 1983; 3: 40-44.

8 Alhalel A, Glovinsky Y, Treister G, Bartov E, Blumenthal M, Belkin M. Long-term follow up of accidental parafoveal laser burns. Retina 1993; 13(2): 152-154.

9 Sasahara M, Noami S, Takahashi M, Honda Y. Optical coherence tomographic observations before and after macular hole formation secondary to laser injury. Am J Ophthalmol 2003; 136(6): 1167-1170.

10 Sou R, Kusaka S, Ohji M, Gomi F, Ikuno Y, Tano Y. Optical coherence tomographic evaluation of a surgically treated traumatic macular hole secondary to Nd:YAG laser injury. Am J Ophthalmol 2003; 135(4): 537-539.

11 Lee GD, Baumal CR, Lally D, Pitcher JD, Vander J, Duker JS. Retinal injury after inadvertent handheld laser exposure. Retina 2014; 34(12): 2388-2396.

12 Sakaguchi H, Ohji M, Kubota A, Otori Y, Hayashi A, Kusaka $S$ et al. Amsler grid examination and optical coherence tomography of a macular hole caused by accidental Nd:YAG laser injury. Am J Ophthalmol 2000; 130(3): 355-356.

13 Fernandez MP, Modi YS, John VJ, Berrocal AM. Accidental Nd:YAG laser-induced macular hole in a pediatric patient. Ophthalmic Surg Lasers Imaging Retina 2013; 44 Online(6): e7-10.

14 Chuang LH, Lai CC, Yang KJ, Chen TL, Ku WC. A traumatic macular hole secondary to a high-energy Nd:YAG laser. Ophthalmic Surg Lasers 2001; 32(1): 73-76.

15 Zamir E, Chowers I. Concerns about laser pointers and macular damage. Arch Ophthalmol 2001; 119(11): 1731-1732.

16 Thach AB, Lopez PF, Snady-McCoy LC, Golub BM, Frambach DA. Accidental Nd:YAG laser injuries to the macula. Am J Ophthalmol 1995; 119(6): 767-773.

17 Newman DK, Flanagan DW. Spontaneous closure of a macular hole secondary to an accidental laser injury. Br J Ophthalmol 2000; 84(9): 1075.

18 Alsulaiman SM, Alrushood AA, Almasaud J, Alkharashi AS, Alzahrani Y, Abboud EB et al. Full-thickness macular hole 
secondary to high-power handheld blue laser: natural history and management outcomes. Am J Ophthalmol 2015; 160(1): 107-113.

19 Potthöfer S, Foerster MH. Vitrectomy and autologous thrombocyte adhesion of an accidental macular hole caused by Nd:YAG laser. Br J Ophthalmol 1997; 81(9): 803-804.

20 Park DH, Kim IT. A case of accidental macular injury by Nd: YAG laser and subsequent 6 year follow-up. Korean J Ophthalmol 2009; 23(3): 207-209.

21 Bará S, Robles M, Tejelo I, Marzoa RI, González H. Green laser pointers for visual astronomy: how much power is enough? Optom Vis Sci 2010; 87(2): 140-144.

22 Chen YJ. Vitrectomy and microperimetry of an accidental macular hole caused by Nd:YAG laser. Case Rep Ophthalmol 2010; 1(2): 80-84.
23 Turaka K, Bryan JS, Gordon AJ, Reddy R, Kwong Jr HM, Sell CH. Laser pointer induced macular damage: case report and mini review. Int Ophthalmol 2012; 32(3): 293-297.

24 Dirani A, Chelala E, Fadlallah A, Antonios R, Cherfan G. Bilateral macular injury from a green laser pointer. Clin Ophthalmol 2013; 7: 2127-2130.

25 Raoof N, Chan TK, Rogers NK, Abdullah W, Haq I, Kelly SP et al. 'Toy' laser macular burns in children. Eye 2014; 28(2): 231-234.

26 Petrou P, Patwary S, Banerjee PJ, Kirkby GR. Bilateral macular hole from a handheld laser pointer. Lancet 2014; 383(9930): 1780.

27 Dhoot DS, Xu D, Srivastava S. High-powered laser pointer injury resulting in macular hole formation. J Pediatr 2014; 164(3): 668.e1. 